Original Paper http://ajol.info/index.php/ijbcs http://indexmedicus.afro.who.int

\title{
Evaluation de l'efficacité de nouveaux programmes de protection phytosanitaire contre les principaux ravageurs du cotonnier Gossypium hirsutum L. au Sénégal
}

\author{
Mokho SARR $^{1,2^{*}}$, Djibril BADIANE ${ }^{2}$ et Banna SANE ${ }^{1,2}$ \\ ${ }^{I}$ Département de Biologie Animale, Faculté des Sciences de Dakar, UCAD, BP 5005, Dakar, Sénégal. \\ ${ }^{2}$ Institut Sénégalais de Recherches Agricoles, Centre de Recherches Zootechniques BP 53 Kolda, Sénégal. \\ *Auteur correspondant ; E-mail : mokho20@hotmail.com
}

\section{RESUME}

Le cotonnier (Gossypium hirsutum L.), deuxième culture de rente au Sénégal, fournit d'importantes ressources financières à la population rurale. Il est exposé à diverses attaques parasitaires essentiellement occasionnées par les chenilles carpophages (Helicoverpa armigera et Earias spp) et les insectes piqueurs suceurs (Bemisia tabaci, Aphis gossypii et Jacobiella fascialis). Les dégâts conduisent à des pertes considérables de rendements en coton-graine, atteignant 50 à $70 \%$, en absence de protection chimique. L'objectif de cette étude est d'évaluer l'efficacité de trois nouveaux programmes de protection phytosanitaire contre ces principaux ravageurs en zones sèche et humide du bassin cotonnier. En plus d'un témoin vulgarisé (PV) et d'un témoin non traité, ces trois programmes innovants, qui diffèrent selon la composition et le positionnement de matières actives, ont été testés dans un dispositif aléatoire complet. Les résultats de l'analyse de la variance (ANOVA) révèlent un bon contrôle des principaux ravageurs par les différents programmes. Les innovations sont plus efficaces que le PV en zone humide, pour le contrôle des carpophages et en zone sèche, pour le contrôle des piqueurs suceurs. Les innovations peuvent être utilisées comme alternatives au PV pour gérer une éventuelle résistance des ravageurs selon la zone.

(C) 2016 International Formulae Group. All rights reserved.

Mots clés: Gossypium hirsutum, ravageurs, protection phytosanitaire, matières actives, Sénégal.

\section{Assessment of new phytosanitary protection programs effectiveness against main cotton plant pests, Gossypium hirsutum L. in Senegal}

\begin{abstract}
Cotton (Gossypium hirsutum L.), second cash crop in Senegal, provides important financial resource for rural population. It is exposed to various pest attacks mainly caused by caterpillars fruit-eating (Helicoverpa armigera and Earias spp) and sucking insects (Bemisia tabaci Aphis gossypii, Jacobiella fascialis). Damage can involve 50 to $70 \%$ yield losses. The objective of this study is to evaluate three new cotton protection programs in dry and wet rainfall conditions on cotton area. These programs were compared with reference witness (PV) and untreated witness in a randomized complete blocks. The innovative programs differ according the composition and positioning of the active matters which compose them. Results of analysis of
\end{abstract}


variance (ANOVA) showed a good control of major pests by the different programs. Innovations are more effective than PV to control caterpillars in wet rainfall conditions and for control of sucking insects in dry rainfall conditions. Innovations can be used as alternative to PV in integrated pest management against an eventual pest resistance.

(C) 2016 International Formulae Group. All rights reserved.

Keywords: Gossypium hirsutum, pests, cotton protection, active matters, Senegal.

\section{INTRODUCTION}

Au Sénégal, le coton représente $5 \%$ des exportations et contribue à $2 \%$ du PIB national (Gueye et al., 2005). La zone de culture du coton couvre $46 \%$ du territoire national et abrite $27 \%$ de la population totale du pays (SODEFITEX, 2009).

Toutefois, le cotonnier est l'une des plantes qui héberge plus de bioagresseurs au monde (Miranda et al., 2013). Outre les adventices et les pathogènes (champignons, bactéries et virus), près de 500 espèces d'insectes, acariens, nématodes et myriapodes identifiées en Afrique vivent au dépend du cotonnier (Celini, 2001 ; Lawson, 2008).

Pour faire face aux contraintes liées aux nuisibles, beaucoup d'efforts ont été entrepris pour réduire leurs dégâts, notamment avec les méthodes de contrôle et les programmes de protection mis au point par la recherche et le développement. La lutte chimique, raisonnée par la gestion sur seuil et le positionnement de matières actives est actuellement la méthode la plus utilisée (Ferron et al., 2006; Agbohessi et al., 2012 ; Badiane et al., 2015). Ainsi, l'élaboration de nouveaux programmes de protection phytosanitaire a été motivée par l'apparition puis le développement de résistance, aux pyréthrinoïdes, de la chenille des capsules Helicoverpa armigera (Hübner), ravageur le plus redoutable sur le cotonnier (Brévaultet al., 2002 ;Traoré, 2008).

C'est dans ce cadre que s'inscrit la présente étude dont l'objectif est d'évaluer l'efficacité de trois nouveaux programmes de protection phytosanitaire, dans le but d'élaborer des stratégies de gestion de la résistance pour une meilleure maîtrise des ravageurs.

\section{MATERIEL ET METHODES Sites d'étude}

L'étude a été menée dans le bassin cotonnier sénégalais, situé dans le tiers Sud du pays. Il est subdivisé en fonction de la pluviométrie en trois zones: une zone sèche (Nord), une zone médiane et une zone humide (Sud) (Figure 1). Toutefois les essais ont été menés en 2012 et 2013 dans des antennes multi locales d'expérimentations (AMEX) de la zone sèche et humide du bassin cotonnier qui sont les plus menacées par les infestations parasitaires. Il s'agit de :

- l'AMEX de Koussanar, dans la région de Tambacounda $\left(13^{\circ} 52^{\prime}\right.$ de latitude Nord et à $14^{\circ} 05^{\prime}$ de longitude Ouest). Cette AMEX se situe en zone sèche du bassin cotonnier. Le climat est de type soudano sahélien et la pluviométrie annuelle varie entre 600 et $700 \mathrm{~mm}$.

- l'AMEX de Syllacounda, dans la région de Kédougou $\left(12^{\circ} 33^{\prime}\right.$ Nord, $12^{\circ} 11^{\prime}$ Ouest). Ce site se trouve dans la zone humide du bassin cotonnier, avec un climat soudanoguinéen recevant entre 800 et $1200 \mathrm{~mm}$ de pluie par an.

\section{Matériel végétal}

La variété de coton utilisée est STAM 129 A, actuellement vulgarisée dans la zone cotonnière sénégalaise. Elle est caractérisée par de longues fibres de couleur blanche et un bon rendement fibre (46\%) (CORAF/WECARD, 2013).

\section{Facteurs étudiés et dispositif expérimental}

Cinq (5) facteurs sont étudiés

(Tableau 1) à savoir: un témoin non traité (NT) ; un témoin vulgarisé (PV) ; innovation 
1 (INNOV 1); innovation 2 (INNOV 2) et innovation 3 (INNOV 3).

Le dispositif expérimental est celui en blocs aléatoires complets avec 4 répétitions. Chaque parcelle élémentaire comprend dix (10) lignes de cotonniers de dix (10) mètres dont les six (6) centrales sont traitées. Une allée large de deux (2) m sépare les blocs (répétitions).

\section{Condition expérimental}

Pour chaque programme, six (6) applications foliaires ont été effectuées (Tableau 1). Ces applications sont démarrées à partir du $31^{\text {ème }}$ jour après la levée des cotonniers, à intervalle de 14 jours à l'exception du programme vulgarisé où le traitement avec Callifan Extra suit un intervalle de temps de 10 jours. L'appareil «Matabi » débitant 60-150 litres de bouillie/ha a permis de faire les applications insecticides. Les différentes matières actives utilisées dans chaque programme ont été définies dans le Tableau 2.

Le traitement de semence a été fait avec Cruiser Extra (Thiamethoxam (350 $\mathrm{g} / \mathrm{l})$, Fludioxonil (8,3 g/l) et Metalaxyl (33 $\mathrm{g} / 1))$; l'herbicidage avec Califor G (Fluométuron (250 g/l), Prométryne (250 $\mathrm{g} / \mathrm{l})$ et Glyphosate $(60 \mathrm{~g} / \mathrm{l}))$ et la fertilisation avec la formule NPKSB (14-23-14 + 5S + 1B). Ces produits sont ceux qui sont vulgarisés au niveau du bassin cotonnier.

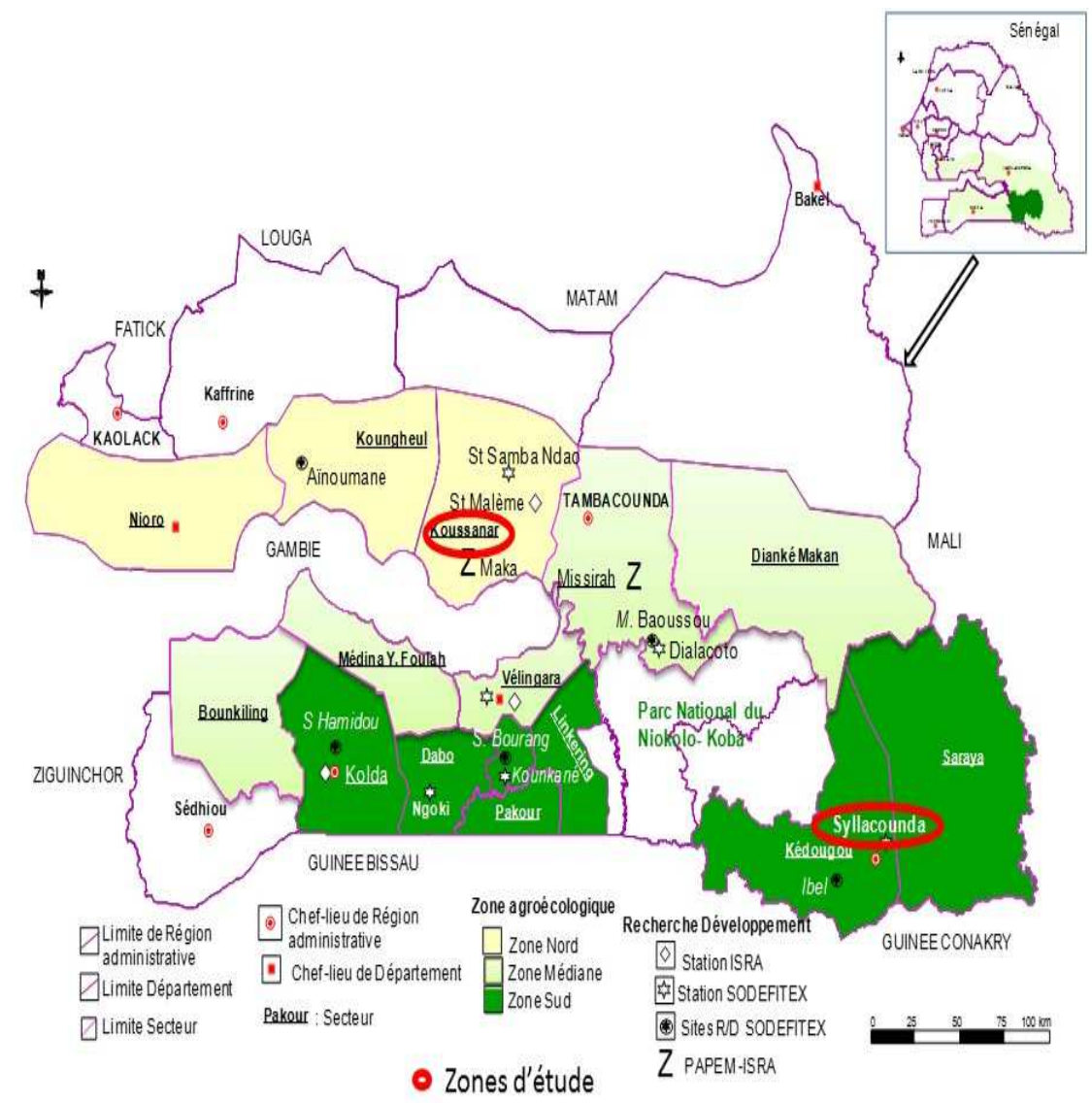

Figure 1 : Localisation du bassin cotonnier sénégalais. Source : SODEFITEX (2009). 


\section{Observation et mesure}

Des relevés parasitaires directs ont été réalisés, chaque semaine, du $30^{\text {ème }}$ au $122^{\text {ème }}$ jour après la levée (JAL) des cotonniers. Ces relevés ont été effectués sur 30 plants par parcelle élémentaire. Les plants ont été pris par groupe de 5 de façon consécutive sur les 6 lignes centrales, selon la méthode de la diagonale (Vayssaire, 1982). Les observations suivantes ont été faites sur l'ensemble des parcelles élémentaires selon les groupes de ravageurs:

- les chenilles carpophages (Helicoverpa armigera, Earias spp): le plant a été examiné en entier et le nombre de chenilles pour chacune des espèces a été compté.

- les insectes piqueur-suceurs (Bemisia tabaci, Jacobielle fascialis et Aphis gossypii) : pour ce groupe de ravageurs, le nombre de plants infestés a été compté. Ainsi, le plant a été considéré attaqué si l'une des 5 feuilles terminales porte au moins une larve et/ou un symptôme d'attaque.

\section{Analyse des données}

Les données recueillies ont fait l'objet d'une analyse de la variance (ANOVA). La comparaison de moyenne a été faite à l'aide du test de Newman Keuls au seuil de 5\%. Les analyses ont été réalisées avec le logiciel GenStat Discovery 10.3.0.0 (Payne et al., 2007).

\section{RESULTATS}

\section{L'effet des programmes sur les carpophages}

Les infestations de $H$. armigera (chenille des capsules) sont plus importantes en zone humide (Syllacounda) qu'en zone sèche (Koussanar). En effet, le seuil de nuisibilité économique (3 chenilles/30 plants) a été atteint dans cette zone (Tableau 3). Quel que soit le site et l'année, les innovations ont permis de réduire significativement ces infestations ( $\mathrm{P}<0,001)$. Toutefois, aucune différence significative n'est notée entre les innovations et le programme vulgarisé en zone sèche. En zone humide, les programmes innovants ont été significativement plus efficaces que le programme vulgarisé en 2013. Par contre en 2012, seule l'innovation 1 s'est révélée plus efficace.

La pression de Earias spp (chenille épineuse) a été relativement faible durant les deux années d'études sur les deux sites (Tableau 4). Le seuil de nuisibilité économique (5 chenilles/30 plants) n'est pas atteint. Les applications insecticides ont permis de contrôler les populations du ravageur $(\mathrm{P}<0,001)$. Toutefois, aucune différence significative n'est observée entre les programmes comparés en 2012. En 2013, les innovations se sont montrées statistiquement plus efficaces que le programme vulgarisé en zone humide.

\section{Effet des programmes sur les piqueurs suceurs}

La présence des piqueurs suceurs est dominée par B. tabaci (mouche blanche) et $A$. gossypii (puceron) qui atteignent le seuil de nuisibilité dans tous les sites (10 plant infestés/30).

Quel que soit le site et l'année, les innovations et le programme vulgarisé (PV) réduisent significativement les infestations des piqueurs-suceurs $(\mathrm{P}<0,001)$.

Toutefois, en zone sèche, les innovations sont statistiquement supérieures au programme vulgarisé en 2013 pour le contrôle de B. tabaci, de J. fascialis et de A. gossypii (Tableaux 5,6 et 7).

En zone humide, seule l'innovation 3 a été plus efficace que le programme vulgarisé pour le contrôle de B. tabaci en 2012. 
Tableau 1 : Programmes comparés.

\begin{tabular}{|c|c|c|c|c|c|c|}
\hline Programmes & AF1 (31 ${ }^{\mathrm{e}}$ JAL) & AF2 $\left(45^{\mathrm{e}} \mathrm{JAL}\right)$ & $\operatorname{AF3}\left(60^{\mathrm{e}} \mathrm{JAL}\right)$ & AF4 $\left(75^{\mathrm{e}}\right.$ JAL $)$ & AF5 (90 JAL) & AF6 (105 JAL) \\
\hline NT & - & - & - & - & - & - \\
\hline PV & Emacot & Emacot & Lambdacal & Lambdacal & Callifan Extra & Callifan Extra \\
\hline INNOV 1 & Tihan & Tihan & Lambdacal & Cobra & Cobra & Thunder \\
\hline INNOV 2 & Calfos & Calfos & Novac & Novac & Lambdacal & Lambdacal \\
\hline INNOV 3 & Tihan & Tihan & Lambdacal & Lambdacal & Emapyr & Emapyr \\
\hline
\end{tabular}

$\overline{A F 1, A F 2, A F 3, A F 4, A F 5, ~ A F 6 ~(a p p l i c a t i o n ~ f o l i a i r e 1, ~ 2, ~ 3, ~ 4,5 ~ e t ~ 6), ~ J A L ~=~ J o u r ~ A p r e ̀ s ~ l a ~ L e v e ́ e ~ d e s ~ c o t o n n i e r s, ~ N T=~ p a r c e l l e ~ n o n ~ t r a i t e ́ e ~(t e ́ m o i n ~ a b s o l u e) ; ~ P V=~ p r o g r a m m e ~ v u l g a r i s e ́ ~ ; ~}$ INNOV= innovation.

Tableau 2: Les différentes matières actives utilisées.

\begin{tabular}{|c|c|c|c|c|c|}
\hline Matière active (g/l) & Nom commercial & $\mathrm{DL}_{50}(\mathrm{mg} / \mathrm{kg})$ & Cible & Famille chimique & Référence \\
\hline Profenofos 500 g/l & CALFOS & 1500 & $\begin{array}{c}\text { carpophages }+ \\
\text { piqueurs suceurs }\end{array}$ & Organophosphorés & $\begin{array}{l}\text { Koriko, } 2010 ; \\
\text { Calliope, } 2014\end{array}$ \\
\hline $\begin{array}{l}\text { Spinétorame }(56 \text { g/l }) / \\
\text { Acétamipride }(64 \text { g/l) }\end{array}$ & COBRA $120 \mathrm{EC}$ & $\begin{array}{c}500 \\
314-417\end{array}$ & $\begin{array}{c}\text { carpophage } \\
\text { piqueurs suceurs }\end{array}$ & $\begin{array}{c}\text { Spinosyne }+ \\
\text { Néonicotinoïde }\end{array}$ & $\begin{array}{c}\text { Couteux et Lejeune, } \\
2012\end{array}$ \\
\hline Emamectinebenzoate (20 g/l) / & EMACOT $20 \mathrm{EC}$ & 208 & carpophage & Avermectine & $\begin{array}{c}\text { Couteux et Lejeune, } \\
2012\end{array}$ \\
\hline $\begin{array}{l}\text { Emamectinebenzoate }(20 \mathrm{~g} / \mathrm{l}) / \\
\text { Pyriproxifène }(60 \mathrm{~g} / \mathrm{l})\end{array}$ & EMAPYR & $\begin{array}{c}208 \\
5000\end{array}$ & $\begin{array}{c}\text { carpophage } \\
\text { piqueurs suceurs }\end{array}$ & $\begin{array}{c}\text { Avermectine }+ \\
\text { Dérivé de pyridine }\end{array}$ & $\begin{array}{l}\text { Couteux et Lejeune, } \\
2012\end{array}$ \\
\hline $\begin{array}{l}\text { Lambda-cyhalothrine (45 g/ha) } \\
\text { Profénofos }(600 \mathrm{~g} / \mathrm{ha})\end{array}$ & LAMDACAL 645 & $\begin{array}{c}56-79 \\
1500\end{array}$ & $\begin{array}{l}\text { Carpophage/pq } \\
\text { piqueurs } \\
\text { suceurs/carpo }\end{array}$ & $\begin{array}{l}\text { Pyréthrinoïdes + } \\
\text { Organophosphorés }\end{array}$ & $\begin{array}{l}\text { Couteux et Lejeune, } \\
2012 \text {; Koriko, } 2010 ; \\
\text { Calliope, } 2014\end{array}$ \\
\hline $\begin{array}{l}\text { Novaluron }(100 \mathrm{~g} / \mathrm{l}) / \\
\text { Acétammipride }(16 \mathrm{~g} / \mathrm{l})\end{array}$ & NOVAC 116 EC & $\begin{array}{c}5000 \\
314-417\end{array}$ & $\begin{array}{c}\text { carpophage } \\
\text { piqueurs suceurs }\end{array}$ & $\begin{array}{l}\text { Benzoyluré + } \\
\text { Néonicotinoïde }\end{array}$ & $\begin{array}{c}\text { BCPC, } 2001 \\
\text { Couteux et Lejeune, }\end{array}$ \\
\hline
\end{tabular}


M. SARR et al. / Int. J. Biol. Chem. Sci. 10(5): 2163-2174, 2016

\begin{tabular}{|c|c|c|c|c|c|}
\hline $\begin{array}{l}\text { Flubendiamide (100) / } \\
\text { Spirotétramate (75 g/l) }\end{array}$ & $\begin{array}{l}\text { TIHAN } 175 \text { O- } \\
\text { TEQ }\end{array}$ & $\begin{array}{l}5000 \\
5000\end{array}$ & $\begin{array}{l}\text { Carpophage/pq } \\
\text { piqueurs } \\
\text { suceurs/carpo }\end{array}$ & $\begin{array}{c}\text { Benzenedicarboxamide } \\
+ \\
\text { Acide tetronic }\end{array}$ & $\begin{array}{l}\text { Agbohessi et al } 2012 \text {; } \\
\text { Bayer, } 2014\end{array}$ \\
\hline $\begin{array}{l}\text { Bétacyfluthrine }(45 \mathrm{~g} / \mathrm{l}) \text { / } \\
\text { Imidaclopride }(100 \mathrm{~g} / \mathrm{l})\end{array}$ & $\begin{array}{l}\text { THUNDER } 145 \\
\text { OTEG }\end{array}$ & $\begin{array}{l}380-551 \\
424-450\end{array}$ & $\begin{array}{c}\text { carpophage } \\
\text { piqueurs suceurs }\end{array}$ & $\begin{array}{l}\text { Pyréthrinoïdes + } \\
\text { Néonicotinoïde }\end{array}$ & $\begin{array}{c}\text { Couteux et Lejeune, } \\
2012\end{array}$ \\
\hline $\begin{array}{l}\text { Bifenthrine (120 g/l) / } \\
\text { Acétammipride (32) / }\end{array}$ & $\begin{array}{l}\text { CALLIFAN } \\
\text { EXTRA } 152 \text { EC }\end{array}$ & $\begin{array}{c}54,5 \\
314-417\end{array}$ & $\begin{array}{c}\text { carpophage } \\
\text { piqueurs suceurs }\end{array}$ & $\begin{array}{l}\text { Pyréthrinoïde + } \\
\text { Néonicotinoïde }\end{array}$ & $\begin{array}{c}\text { Couteux et Lejeune, } \\
2012\end{array}$ \\
\hline
\end{tabular}

DL50= Dose létale 50= quantité de matière, administrée en une seule fois, qui cause la mort de 50 \% (la moitié) d'un groupe d'animaux d'essai, carpo= carpophage ; pq= piqueurs suceurs.

Tableau 3 : Niveaux moyens annuels de Helicoverpa armigera en fonction des programmes.

\begin{tabular}{|c|c|c|c|c|}
\hline \multirow{3}{*}{ Traitement } & \multicolumn{4}{|c|}{ Nombre de chenilles $H$. armigera sur 30 plants } \\
\hline & \multicolumn{2}{|c|}{ Koussanar } & \multicolumn{2}{|c|}{ Syllacounda } \\
\hline & 2012 & 2013 & 2012 & 2013 \\
\hline NT & $1,45 \pm 0,15^{\mathrm{b}}$ & $3,04 \pm 0,46^{\mathrm{b}}$ & $3,03 \pm 0,24^{\mathrm{c}}$ & $3,19 \pm 0,21^{c}$ \\
\hline PV & $0,20 \pm 0,10^{\mathrm{a}}$ & $0,04 \pm 0,04^{\mathrm{a}}$ & $0,53 \pm 0,12^{b}$ & $0,86 \pm 0,15^{b}$ \\
\hline INNOV 1 & $0,25 \pm 0,10^{\mathrm{a}}$ & $0,00 \pm 0,00^{\mathrm{a}}$ & $0,10 \pm 0,05^{\mathrm{a}}$ & $0,33 \pm 0,09^{\circ}$ \\
\hline INNOV 2 & $0,08 \pm 0,05^{\mathrm{a}}$ & $0,04 \pm 0,04^{\mathrm{a}}$ & $0,39 \pm 0,13^{a b}$ & $0,47 \pm 0,11^{\circ}$ \\
\hline INNOV 3 & $0,16 \pm 0,07^{\mathrm{a}}$ & $0,25 \pm 0,10^{\mathrm{a}}$ & $0,60 \pm 0,11^{\mathrm{b}}$ & $0,27 \pm 0,07^{\circ}$ \\
\hline Moyenne \pm écart type $(n=4)$ & $0,43 \pm 0,06$ & $0,67 \pm 0,14$ & $0,93 \pm 0,11$ & $1,03 \pm 0,10$ \\
\hline Probabilité et signification & $<0,001 * * *$ & $<0,001 * * *$ & $<0,001 * * *$ & $<0,001 * * *$ \\
\hline
\end{tabular}

Les moyennes affectées de la même lettre « $a, b$, ab» constituent statistiquement un groupe homogène. Chaque groupe homogène présente une différence hautement significative (***) avec les autres groupe ; NT= parcelle non traitée (témoin absolue); $P V=$ programme vulgarisé ; INNOV= innovation. 
M. SARR et al. / Int. J. Biol. Chem. Sci. 10(5): 2163-2174, 2016

Tableau 4 : Niveaux moyens annuels d'Earias spp (chenille épineuse) en fonction des programmes.

\begin{tabular}{|c|c|c|c|c|}
\hline \multirow{3}{*}{ Traitement } & \multicolumn{4}{|c|}{ Nombre de chenilles épineuses sur 30 plants } \\
\hline & \multicolumn{2}{|c|}{ Koussanar } & \multicolumn{2}{|c|}{ Syllacounda } \\
\hline & 2012 & 2013 & 2012 & 2013 \\
\hline NT & $0,92 \pm 0,12^{b}$ & $0,90 \pm 0,16^{\mathrm{b}}$ & $3,25 \pm 0,37^{b}$ & $2,80 \pm 0,21^{\mathrm{c}}$ \\
\hline PV & $0,00 \pm 0,00^{a}$ & $0,10 \pm 0,00^{\mathrm{a}}$ & $0,39 \pm 0,14^{\mathrm{a}}$ & $0,92 \pm 0,17^{b}$ \\
\hline INNOV 1 & $0,10 \pm 0,05^{a}$ & $0,00 \pm 0,00^{\mathrm{a}}$ & $0,21 \pm 0,07^{\mathrm{a}}$ & $0,50 \pm 0,09^{\mathrm{a}}$ \\
\hline INNOV 2 & $0,00 \pm 0,00^{\mathrm{a}}$ & $0,00 \pm 0,00^{\mathrm{a}}$ & $0,21 \pm 0,07^{\mathrm{a}}$ & $0,47 \pm 0,09^{\mathrm{a}}$ \\
\hline INNOV 3 & $0,03 \pm 0,03^{a}$ & $0,00 \pm 0,00^{\mathrm{a}}$ & $0,39 \pm 0,15^{\mathrm{a}}$ & $0,16 \pm 0,06^{\mathrm{a}}$ \\
\hline Moyenne \pm écart type $(n=4)$ & $0,21 \pm 0,04$ & $0,20 \pm 0,05$ & $0,89 \pm 0,13$ & $0,97 \pm 0,09$ \\
\hline Probabilité et signification & $<0,001 * * *$ & $<0,001 * * *$ & $<0,001 * * *$ & $<0,001 * * *$ \\
\hline
\end{tabular}

Les moyennes affectées de la même lettre " $a, b, a b$ » constituent statistiquement un groupe homogène. Chaque groupe homogène présente une différence hautement significative (***) avec les autres groupe; $N T=$ parcelle non traitée (témoin absolue); $P V=$ programme vulgarisé ; INNOV= innovation.

Tableau 5: Niveaux moyens annuels de Bemisia tabaci (mouche blanche) en fonction des programmes.

\begin{tabular}{|c|c|c|c|c|}
\hline \multirow{3}{*}{ Traitement } & \multicolumn{4}{|c|}{ Nombre de plants infestés par les mouches blanches sur 30 plants } \\
\hline & \multicolumn{2}{|c|}{ Koussanar } & \multicolumn{2}{|c|}{$\begin{array}{l}\text { Syllacounda } \\
\end{array}$} \\
\hline & 2012 & 2013 & 2012 & 2013 \\
\hline NT & $8,50 \pm 0,53^{b}$ & $11,90 \pm 0,51^{\mathrm{c}}$ & $9,32 \pm 0,40^{\mathrm{c}}$ & $4,22 \pm 0,28^{\mathrm{b}}$ \\
\hline PV & $3,03 \pm 0,32^{a}$ & $2,03 \pm 0,49^{\mathrm{b}}$ & $4,43 \pm 0,28^{\mathrm{b}}$ & $2,66 \pm 0,22^{a}$ \\
\hline INNOV 1 & $2,42 \pm 0,31^{\mathrm{a}}$ & $0,25 \pm 0,11^{a}$ & $3,78 \pm 0,28^{a b}$ & $2,56 \pm 0,23^{a}$ \\
\hline INNOV 2 & $2,25 \pm 0,34^{\mathrm{a}}$ & $0,07 \pm 0,04^{\mathrm{a}}$ & $3,25 \pm 0,28^{a b}$ & $2,83 \pm 0,26^{\mathrm{a}}$ \\
\hline INNOV 3 & $2,39 \pm 0,42^{a}$ & $0,35 \pm 0,17^{\mathrm{a}}$ & $2,92 \pm 0,30^{\mathrm{a}}$ & $2,52 \pm 0,23^{\mathrm{a}}$ \\
\hline Moyenne \pm écart type $(n=4)$ & $3,72 \pm 0,26$ & $2,92 \pm 0,41$ & $4,74 \pm 0,24$ & $2,96 \pm 0,11$ \\
\hline Probabilité et signification & $<0,001 * * *$ & $<0,001 * * *$ & $<0,001 * * *$ & $<0,001 * * *$ \\
\hline
\end{tabular}

Les moyennes affectées de la même lettre « $a, b, a b »$ constituent statistiquement un groupe homogène. Chaque groupe homogène présente une différence hautement significative (***) avec les autres groupe; $N T=$ parcelle non traitée (témoin absolue); $P V=$ programme vulgarisé $; I N N O V=$ innovation. 
Tableau 6 : Niveaux moyens annuels de Jacobiella fascialis (jasside) en fonction des programmes.

\begin{tabular}{|c|c|c|c|c|}
\hline \multirow{3}{*}{ Traitement } & \multicolumn{4}{|c|}{ Nombre de plants infestés par les jassides sur 30 plants } \\
\hline & \multicolumn{2}{|c|}{ Koussanar } & \multicolumn{2}{|c|}{ Syllacounda } \\
\hline & 2012 & 2013 & 2012 & 2013 \\
\hline NT & $1,71 \pm 0,41^{b}$ & $6,07 \pm 0,61^{\mathrm{c}}$ & $5,96 \pm 0,36^{b}$ & $4,14 \pm 0,25^{b}$ \\
\hline PV & $0,53 \pm 0,20^{a}$ & $1,32 \pm 0,39^{\mathrm{b}}$ & $2,71 \pm 0,26^{\mathrm{a}}$ & $2,54 \pm 0,19^{a}$ \\
\hline INNOV 1 & $0,32 \pm 0,14^{\mathrm{a}}$ & $0,28 \pm 0,16^{\mathrm{a}}$ & $2,57 \pm 0,28^{a}$ & $2,85 \pm 0,25^{\mathrm{a}}$ \\
\hline INNOV 2 & $0,53 \pm 0,26^{a}$ & $0,07 \pm 0,00^{\mathrm{a}}$ & $2,60 \pm 0,18^{a}$ & $2,58 \pm 0,25^{\mathrm{a}}$ \\
\hline INNOV 3 & $0,28 \pm 0,13^{a}$ & $0,03 \pm 0,03^{\mathrm{a}}$ & $1,93 \pm 0,19^{a}$ & $2,93 \pm 0,28^{\mathrm{a}}$ \\
\hline Moyenne \pm écart type $(n=4)$ & $0,68 \pm 0,12$ & $1,56 \pm 0,24$ & $3,16 \pm 0,16$ & $3,01 \pm 0,11$ \\
\hline Probabilité et signification & $<0,001 * * *$ & $<0,001 * * *$ & $<0,001 * * *$ & $<0,001 * * *$ \\
\hline
\end{tabular}

Les moyennes affectées de la même lettre « $a, b, a b »$ constituent statistiquement un groupe homogène. Chaque groupe homogène présente une différence hautement significative (***) avec les autres groupe; $N T=$ parcelle non traitée (témoin absolue); $P V=$ programme vulgarisé ; INNOV= innovation.

Tableau 7 : Niveaux moyens annuels de Aphis gossypii (puceron) en fonction des programmes.

\begin{tabular}{|c|c|c|c|c|}
\hline \multirow{3}{*}{ Traitement } & \multicolumn{4}{|c|}{ Nombre de plants infestés par les pucerons sur 30 plants } \\
\hline & \multicolumn{2}{|c|}{ Koussanar } & \multicolumn{2}{|c|}{$\begin{array}{c}\text { Syllacounda } \\
\end{array}$} \\
\hline & 2012 & 2013 & 2012 & 2013 \\
\hline NT & $10,10 \pm 0,66^{\mathrm{c}}$ & $10,00 \pm 0,68^{\mathrm{c}}$ & $23,00 \pm 1,25^{b}$ & $22,35 \pm 1,33^{b}$ \\
\hline PV & $4,89 \pm 0,50^{\mathrm{b}}$ & $1,96 \pm 0,45^{\mathrm{b}}$ & $14,00 \pm 1,54^{\mathrm{a}}$ & $11,83 \pm 1,11^{\mathrm{a}}$ \\
\hline INNOV 1 & $3,10 \pm 0,51^{\mathrm{a}}$ & $0,14 \pm 0,11^{\mathrm{a}}$ & $12,68 \pm 1,14^{\mathrm{a}}$ & $9,87 \pm 0,69^{\mathrm{a}}$ \\
\hline INNOV 2 & $3,28 \pm 0,41^{\mathrm{a}}$ & $0,21 \pm 0,15^{\mathrm{a}}$ & $12,71 \pm 1,55^{\mathrm{a}}$ & $9,73 \pm 0,73^{\mathrm{a}}$ \\
\hline INNOV 3 & $2,89 \pm 0,40^{a}$ & $0,10 \pm 0,00^{\mathrm{a}}$ & $12,14 \pm 1,50^{\mathrm{a}}$ & $9,77 \pm 0,67^{\mathrm{a}}$ \\
\hline Moyenne \pm écart type $(n=4)$ & $4,86 \pm 0,32$ & $2,48 \pm 0,36$ & $15,49 \pm 0,76$ & $12,71 \pm 0,52$ \\
\hline Probabilité et signification & $<0,001 * * *$ & $<0,001 * * *$ & $<0,001 * * *$ & $<0,001 * * *$ \\
\hline
\end{tabular}

Les moyennes affectées de la même lettre « $a, b, a b »$ constituent statistiquement un groupe homogène. Chaque groupe homogène présente une différence hautement significative (***) avec les autres groupe; $N T=$ parcelle non traitée (témoin absolue); $P V=$ programme vulgarisé $; I N N O V=$ innovation. 


\section{DISCUSSION}

Les résultats obtenus au cours des deux années d'étude indiquent que les programmes innovants sont efficaces pour le contrôle des principaux ravageurs du cotonnier. Ces programmes affichent quasiment le même niveau d'efficacité. Ainsi les niveaux très faibles de $H$. armigera, dans les innovations comparés à celui du témoin absolu $(\mathrm{NT}=$ non traité) et du programme vulgarisé, montrent que les traitements insecticides utilisés contre ce ravageur permettent de le maintenir à des niveaux inférieurs au seuil de nuisibilité (3 individus/30 plants).

Les attaques faibles voire l'absence de Earias spp dans les innovations, révèlent que les programmes innovants ont permis aussi de contrôler ce ravageur. Ainsi dans nos conditions d'expérimentation nous pouvons dire que les matières actives utilisées contre les carpophages (H. armigera et Earias spp), en l'occurrence Flubendiamide, Spirotétramate, Spinétorame, Bétacyfluthrine, Profenofos, Novaluron, Lambda-cyhalothrine, réduisent leur pression à des niveaux d'infestation faibles (inférieurs au seuil). Ces résultats corroborent ceux de Badji (2010) qui a montré que Profenofos, Flubendiamide, Spirotétramate ont un contrôle efficace sur $H$. armigera et Earias spp.

Les programmes innovants semblent être au même degré d'efficacité que le programme vulgarisé en zone sèche, mais ils seraient plus efficaces que le PV en zone humide. Selon certains auteurs (Badiane, 2008 ; Diop, 2013), la période critique des carpophages dans le bassin cotonnier sénégalais se situe entre le $60^{\mathrm{e}}$ et le $90^{\mathrm{e}}$ jour après la levée des cotonniers. Ce qui nous laisse penser que les applications effectuées à cette période (AF3, AF4, AF5) ont permis de mettre en évidence l'effet alternatif des nouveaux programmes de protection. En effet dans le programme vulgarisé, des pyréthrinoïdes (Lambda-cyhalothrine, Bifenthrine) ont été majoritairement utilisés à ces dates, alors que dans les innovations les pyréthrinoïdes sont alternés avec des spinosynes (Spinétorame) dans l'innovation 1, des Benzoylurées (Novaluron) dans l'innovation 2, et des avermectines (Emamectine benzoate) dans l'innovation 3 . Ce qui expliquerait que le positionnement de ces matières actives préconisé dans les nouveaux programmes permet de casser la résistance des ravageurs et d'atteindre plus facilement la cible (carpophages). L'efficacité des matières actives est mieux assurée en période de forte infestation. Ainsi la forte infestation observée en zone humide nous permet de mettre en exergue cette hypothèse.

Les faibles infestations des piqueurs suceurs (B. tabaci, J. fascialis et A. gossypii) dans les innovations et le programme vulgarisé, comparées à celles des parcelles non traitées révèlent que les traitements insecticides contrôlent les attaques de ces ravageurs. Partant de ces résultats nous pouvons dire que, dans nos conditions d'expérimentation, les matières actives utilisées contre les piqueur-suceurs (Spirotétramate, Profenofos, Acétamipride, Pyriproxène et Imidaclopride) permettent de les contrôler. Ces résultats sont en harmonie avec ceux de Ayeva et al. (2014) qui soulignent l'efficacité de Spirotétramate, Acétamipride, Pyriproxène et Imidaclopride sur B. tabaci et $J$. fascialis. Barrania et AbouTaleb (2014) abondent dans le même sens et ont montré l'efficacité de Acétamipride et de Imidaclopride pour le contrôle de B. tabaci et de A. gossypii. Selon les mêmes auteurs, Pyriproxène permet aussi de réduire les populations de B. tabaci. Pour Aslam et al. (2004), Acétamipride et Imidaclopride permettent de contrôler J. fascialis.

Les programmes innovants seraient plus efficaces que le $\mathrm{PV}$ en zone sèche. En condition normale de culture (semis dans les délais, pluviométrie régulière), les fortes présences des insectes piqueurs suceurs, particulièrement celle de $B$. tabaci et de $J$. 
fascialis sont notées entre le $60^{\mathrm{e}}$ et $105^{\mathrm{e}}$ jour après la levée des cotonniers (Badiane, 2008 ; Badji, 2010). Les faibles infestations dans les innovations par rapports au programme vulgarisé en zone sèche, peuvent être liées aux traitements effectués à cette période (AF1, AF2, AF3). En effet selon la période d'application, les pyréthrinoïdes sont alternés et associés à une matière active spécifique contre les piqueurs suceurs dans les innovations. Il s'agit de Cobra (Spinétorame/Acétamipride) dans l'innovation 1, de Novac (Novaluron/Acétammipride) dans l'innovation 2 et de Emapyr (Emamectine benzoate/Pyriproxifène) dans l'innovation 3 . Le positionnement en fonction de la pression parasitaires des alternatifs aux pyréthrinoïdes, associés à une matière actives spécifique semble donc avoir un meilleur contrôle des piqueurs suceurs.

Il est à remarquer que les infestations de $A$. gossypii quasiment au seuil ou légèrement audessus du seuil en zone humide (Syllacounda) ont été surtout obtenues en fin de campagne, période pendant laquelle la reproduction d'A. gossypii est intense pour la préparation des migrations vers d'autres spéculations (Celini, 2001 ; Diatta, 2011). Par ailleurs, en zone humide les cotonniers entrent tardivement en phase de senescence d'où la disponibilité de support nutritif pour les piqueurs suceurs, ce qui expliquerait la difficulté de leur contrôle en fin de campagne. Toutefois les programmes innovants semblent être à la hauteur de la gestion de ces ravageurs.

\section{Conclusion}

Au Sénégal, la culture cotonnière fait l'objet de nombreuses attaques parasitaires, qui en absence de protection chimique conduisent à des pertes considérables de rendement pouvant atteindre 50 à $70 \%$. Dans cette étude nous avons évalué l'efficacité de trois nouveaux programmes de protection phytosanitaires, pour le contrôle des principaux ravageurs. Les résultats obtenus montrent que les ravageurs qui causent des soucis majeurs (chenilles carpophages) ont pu être contrôlés par les programmes testés. Il en ressort aussi de ces résultats que les piqueurs suceurs en l'occurrence Bemisia tabaci, Jacobiella fascialis et Aphis gossypii occupant de nos jours une place de plus en plus importante dans le complexe parasitaire sont contrôlés grâce à l'efficacité de ces programmes. Le programme vulgarisé reste toujours efficace. Les innovations pourraient être utilisées comme des alternatives pour prévenir une éventuelle résistance des déprédateurs.

\section{CONFLIT D'INTERETS}

Les auteurs déclarent qu'ils n'ont pas de conflit d'intérêts.

\section{CONTRIBUTIONS DES AUTEURS}

MS, DB et BS: Conception et élaboration des protocoles, expérimentation au champ. MS: Analyse et interprétation des données. MS et DB: Préparation du manuscrit. Tous les auteurs ont lu et approuvé la version finale du manuscrit.

\section{REMERCIEMENTS}

Les auteurs remercient les techniciens des sites d'expérimentation, la Société de Développement des Fibres Textiles (SODEFITEX), la Fédération Nationale des Producteurs de Coton (FNPC) et le Programme Régional de Protection Intégrée du Cotonnier en Afrique (PR-PICA) pour leur collaboration.

\section{REFERENCES}

Agbohessi T, Toko II, Attakpa EY, Kestemo P. 2012. Synthèse des caractéristiques physico-chimiques et effets écotoxicologiques du Tihan 175 OTEQ utilisé dans la protection du cotonnier au Bénin. Int. J. Biol. Chem. Sci., 6(5): 2281-2292. 
Aslam M, Razaq M, Shah SA, Ahmad F. 2004. Comparative efficacy of different insecticides against sucking pests of cotton. J. Res. Sci., 15: 53-58.

Ayeva B, Ochou OG, Togola M, Hema O, Bonni G, Badiane D, Sawadogo F, Ciss I. 2014. Evaluation de l'efficacité de nouvelles matières actives ou association de matières actives. Rapport de la septième réunion-bilan du PR-PICA, Dakar, 28 p.

Badiane D. 2008. Bioécologie de la mouche blanche (Bemisia tabaci) Gennadius (Homoptera, Aleyrodidae) et mise sur place de stratégie de lutte sur le cotonnier (Gossypium hirsutum) au Sénégal. Mémoire de DEA, Production Végétale, agronomie et protection des cultures. Thiès : université de Thiès, 72 p.

Badiane D, Gueye MT, Coly EV, Faye O. 2015. Gestion intégrée des principaux ravageurs du cotonnier au Sénégal et en Afrique occidentale. Int. J. Biol. Chem. Sci., 9(5): 2654-2667.

Badji A. 2010. Gestion des principaux insectes ravageurs du cotonnier au Sénégal. Mémoire de fin de cycle, Ingénieur Agronome, Institut Polytechnique Rural de Formation et de Recherche Appliquée (IPR/IFRA), Katibougou, Mali, 62p.

Barrania AA, Abou-Taleb HK. 2014. Field Efficiency of Some Insecticide Treatments against Whitefly, Bemisia tabaci, Cotton Aphid, Aphis gossypii and Their Associated Predator, Chrysopa vulgaris, in Cotton Plants. Alex. J. Agric. Res., 59(2): 105-111.

Bayer. 2014. Tihan 175. Fiche technique, Monhaim-Allemagne, $4 \mathrm{p}$.

BCPC. 2001. The e-pesticide manual (version 2.0) [logiciel]. Omega Park, Alton, Hampshire, GU34 2QD, UK, Angleterre.
Brévault T, Beyo J, Nibouche S, Vaissayre M. 2002. La résistance des insectes aux insecticides: Problématique et enjeux en Afrique centrale. Actes du colloque Savanes africaines : des espaces en mutation, des acteurs face à de nouveaux défis. CIRAD/PRASAC Garoua, Cameroun, 27 au 31 mai 2002.

Calliope. 2014. Cyperméthrine / Profénofos. Fiche technique, Paris, 1p.

Celini L. 2001. Le Puceron du cotonnier Aphis gossypii (Glover) et son parasite Aphelinus gossypii. Timberlake en République Centrafricaine. Insectes, 122: 7-10.

CORAF/WECARD. 2013. Informations sur les Technologies agricoles du Programme National Coton. Fiche technique, Togo, $1 \mathrm{p}$.

Couteux A, Lejeune V. 2012. Index Phytosanitaire ACTA 2012. ACTA: Paris ; 940 p.

Diatta J. 2011. Mise sur pied d'une stratégie de gestion durable des ravageurs du cotonnier (Gossypium hirsutum L.) au Sénégal. Mémoire de fin de cycle, Productions Végétales, Ingénieur Agronome, Université de Thiès, Ecole Nationale Supérieure d'Agriculture (ENSA), Thiès (Sénégal), 57 p.

Diop J. 2013. Surveillance des ennemis du cotonnier pour une meilleure gestion de la production au Sénégal. Mémoire, Protection durable des cultures et de l'environnement. Centre Régional AGRHYMET, Niamey-Niger, 67 p.

Ferron P, Deguine JP, Mouté JE. 2006. Évolution de la protection phytosanitaire du cotonnier : un cas d'école. Cahiers Agricultures, 15(1): 128-134.

Gueye M, Beye AM, Badiane D. 2005. Le cotonnier. In Bilan de la Recherche Agricole et Agroalimentaire au Sénégal. ISRA-ITA-CIRAD : Dakar ; 189-205. 
Koriko M. 2010. Extraction, caractérisation, réactivité chimique des substances humiques naturelles avec les phosphates de Hahotoe-Kpogame et leurs propriétés photodégradantes. Chimie/physicochimie des matériaux. Thèse en cotutelle, UL et UBPCF, Lomé, 140 p.

Lawson AJ. 2008. Effet de différentes pratiques de taille sur l'amélioration des performances agronomiques du cotonnier Gossypium hirsutum L. [en ligne]. Thèse, Sciences et Techniques de Production Végétale, ingénierie agronome, Université de Parakou, Parakou, $60 \mathrm{p}$.

Miranda JE, Rodrigues SMM, Almeida RP, Sylva CAD, Togola M, Hema SAO, Somé NH, Boni G, Adegnika MO, Doyam AN, Diambo B. 2013. Reconnaissances de ravageurs et ennemies naturels pour les pays C-4. Embrapa, Brésil, 70 p.
Payne RW, Murray DA, Harding SA, Baird DB, Soutar DM. 2007. GenStat for Windows (10th Edition) Introduction. VSN International, Hemel Hempstead.

SODEFITEX (Société de Développement et des Fibres Textiles). 2009. Capitalisation des pratiques en fumures organique en zone cotonnière du Sénégal. Rapport de l'étude APROCA sur les pratiques en fumure organique, $17 \mathrm{p}$.

Traoré O. 2008. Les succès de la lutte intégrée contre les ravageurs du cotonnier en Afrique de l'Ouest. $67^{\text {ème }}$ réunion plénière de l'ICAC. INERA, Burkina Faso, 17 p.

Vayssaire M. 1982. Méthodes d'échantillonnage des populations d'insectes dans les cultures cotonnières d'Afrique. Entomophaga, special : 2529. 